\title{
INCREASING METROLOGICAL AUTONOMY OF IN-PLANT MEASURING SYSTEMS
}

\author{
Mykola Mykyychuk', Roman Kochan ${ }^{2,3}$, Orest Kochan ${ }^{4}$, Su Jun ${ }^{5}$, \\ Bohdan Stadnyk ${ }^{4}$, Petro Stolyarchuk ${ }^{1}$
}

\author{
1 Department of Metrology, Standardization and Certification, Lviv Polytechnic National University, Karpinskyy \\ Str. 1, 79013, Lviv, Ukraine \\ 2 Department of Computer Science and Automatics, University of Bielsko-Biala, Willowa Str. 2, $43-300$ Bielsko- \\ Biała, Poland \\ 3 Department of Specialized Computer Systems, Lviv Politechnic National University, Karpinskyy Str. 1, 79013, \\ Lviv, Ukraine, e-mail: kochan.roman@gmail.com \\ 4 Department of Information-Measuring Engineering, Lviv Polytechnic National University, Karpinskyy Str. 1, \\ 79013, Lviv, Ukraine, e-mail: orestvk@gmail.com \\ 5 School of Computer Science, Hubei University of Technology, Hubei, China
}

Received: 2016.07.18 Accepted: 2016.10.08 Published: 2016.12.01

\begin{abstract}
The authors offer to solve the problem of providing traceability of measurements by increasing metrological autonomy of in-plant measuring systems. The paper shows the expedience of increasing metrological autonomy by creating a "virtual" reference. There are analysed possible variants of implementation of the "virtual" reference, which will provide high metrological stability of measurements at insignificant additional expenses. The authors point out the necessity of creation of universal technical and programmatic means of mutual comparison for the in-plant measuring systems to increase the reliability of measurements in the conditions of metrological autonomy.
\end{abstract}

Keywords: metrological autonomy, in-plant measuring system, measuring equipment, metrological reliability, comparison, calibration.

\section{INTRODUCTION}

The solution of important scientific and technical problems largely depends on the traceability of measurements. In particular, the speed and accuracy of decision making on functioning many industrial processes and systems depends on the accuracy, correctness and timeliness of measurements and monitoring of various parameters. Therefore, the level of measurement reliability in various fields of human activity largely determines their effectiveness $[1,8,11,15,22]$.

The traceability of measurements is referred to as a state of measurement in which results of measurements are expressed in legal units and their errors and uncertainties are known and with a certain probability do not exceed preset limits $[15,19]$.
Foundations of metrological traceability are activities associated with the development and continuous improvement of metrological supervision (MS). MS is the establishment and application of metrological rules and regulations, as well as the development, production and application of technical facilities needed to achieve the desired traceability and accuracy of measurements. To effectively address the issues of MS there are state and departmental metrological services whose goal is to create legal, regulatory, scientific, technical and institutional frameworks to ensure traceability in a state. These goals are achieved by the implementation of specific metrological activities: research, maintenance, verification and certification of standards, calibration of measuring instruments (MI), certification of measurement techniques. 
Because of significant deterioration [12] of standard equipment and rising prices on metrology services, the role of organizational and technical measures to ensure a certain metrological autonomy of in-plant measuring systems that will reduce the cost of their metrological support while ensuring the required level of unity, accuracy and reliability of measurements significantly increases [1]. That is why the market for metrological services is estimated to be about $4 \%$ of GDP in developed countries $[6,7]$. The need for simplification and reduction of prices led to the invention of methods of in-situ testing and calibration [9, 13, 14, 18], however these methods can be applied just under specific conditions. In this paper the authors try to come up with more general approaches that can be applied in wide range of conditions.

Nowadays, one of the promising areas to improve MIs designed for storage, reproduction and transfer of the physical units is creation of new complex references formed on the basis of a group of passive-measuring instruments of physical units [4]. The composition of such a complex includes several highly stable measures, which are equipped with means of verification. Moreover, some elements of the complex do not just perform the function of a "reserve" reference of some basic element, but they are full members of the complex and directly influence the formation of the output unit of the physical quantity of the complex reference [5]. This approach can increase the stability of reproduction of a physical unit of the complex reference compared with the stability of individual elements. Also, creation of the algorithm to process the results of mutual comparisons of the elements of the complex based on the maximum likelihood method can significantly simplify comparisons and improve the efficiency of statistical processing of the data $[4,5]$. Thus, the implementation of the concept of metrological autonomy in modern conditions can increase the metrological stability of in-plant measuring systems.

However, nowadays, the practical implementation of the concept of increasing metrological autonomy of in-plant measurement systems existing in industry and science is not sufficiently developing.

\section{RESEARCH OBJECTIVES}

The purpose of this paper is to study the efficiency of the methods to ensure the measure- ment traceability by implementing the methods for increasing metrological autonomy for various branches of industry and science.

Any measuring process contains two interrelated parts:

- A set of transactions to obtain information about the value of a physical quantity: interaction with the object of measurement, measuring conversion of the measured signals to the form convenient for use and processing of the obtained measuring results;

- A set of transactions to ensure traceability, reproduction, storage and transfer of units of physical quantities, creation of conditions to ensure metrological serviceability of MIs.

These interconnected components of the measuring process determine the quality of measurements in the bottom line. Recently, due to globalization in the world economy, there is a tendency to increase the role of the traceability of measurements. However, increasing the competitiveness of products includes cutting down on expenses of all the components of a production process and, in particular, expenses on the procedures of the metrological service of MIs. One way in this direction is to increase intercalibration time while maintaining the required metrological reliability of MIs. This path involves improving metrological autonomy of measurements.

Metrological autonomy is referred to as a long term ability to maintain metrological characteristics of a MI without use of reference facilities of higher rank (i.e. they are at higher stages of transfer of the unit of a physical quantity [5]).

According to the requirements of [11], organisations have to determine the level of required control measures and establish requirements for managing the measuring system that they are going to use as a part of their overall management system. The effective management of the measuring system has to ensure fitness of measuring equipment and measuring processes for their intended use and therefore plays an important role in achieving the goals of quality processes for which it was created and manage the risk of getting distorted results of measurements.

The main methodological principle of the current system of metrological support is periodic calibration of MIs at regular intervals to confirm their metrological reliability $[8,12]$. Such an approach to ensure measurement traceability does 
not take into account the specific application of the MIs in actual operating conditions, which causes additional errors [17]. These additional errors typically arise from nonconformity of conditions in which metrological confirmation of MIs' reliability is carried out with operating conditions of these MIs $[3,8,17,20]$.

Modern manufacturing processes require more accurate control and reduction of losses due to improper measurements. That is why the requirements to ensure metrological reliability of MIs during intercalibration intervals are increasing $[3,17]$.

One way to increase the unity of mass measurements is to create a "virtual" reference, accuracy of which would define the current level of traceability of measurements in an organization. The praxis of the "virtual" references is known $[10,21]$ and is used to increase the metrological reliability of reproduction of units. However, nowadays, the concept of the "virtual" references has not been studied well for a wide range of industrial measurements, however, state of the art of microelectronics and computer technology will allow producing "virtual" references without difficulties.

Each measuring process uses quite a stable set of MIs, whose the measurement results are used for a long time. This set of MI has metrological characteristics which are in a certain deterministic connection with technological modes, so this set of MIs can be considered as an in-plant measuring system, which is typical for a process. The concept of creating a "virtual" reference is to create techniques and methods that ensure mutual comparing procedures of MIs as well as accumulation and use of information about their individual metrological characteristics.

To implement a "virtual" reference for an inplant measuring system it is obligatory to ensure such properties as metrological efficiency and metrological uniformity. Metrological efficiency can be referred to as the ability of an in-plant measuring system to ensure operational control over metrological characteristics of the MIs used in the in-plant measuring system. When operating together a sufficiently stable set of the MIs in the in-plant measuring system for a long time it is easy enough to create an information database on individual accuracy and stability of each MI and develop a technique for updating the database [13]. Metrological uniformity can be referred to as providing of technical capacity for effective comparisons of the MIs used in the in-plant mea- suring system. State of the art of microelectronics makes it possible to create highly accurate facilities that can provide the necessary technical capabilities at low cost to implement comparing procedures $[8,16]$.

Let's consider a possible realization of the "virtual" reference for widespread in in-plant measuring systems. In the in-plant measuring system (at a separate company or an organization), there is a set of operating MIs that measure process parameters. This set of MIs can be split into several groups of MIs of the same kind. Each such a group measures similar input parameters. Then, for each group of similar MIs, it is possible to create a "virtual" reference of a physical value that is measured within the group. The specifics of creation of a "virtual" reference are in the peculiarities of the procedures of mutual comparisons of MI and statistical operations for determining the physical unit value of the reference.

The method of mutual comparisons within groups of MIs of the same kind can be carried out in the following ways. In case of closely placed MIs, e.g. on a control board, it is necessary to implement the scheme and the schedule of their periodic disconnection from sources of measuring signals. After disconnecting them from the measuring signals a stable reference signal should be simultaneously connected to the inputs of the all MIs. Using the series of readings obtained from this set of MIs of the same kind $-\left\{\mathrm{X}_{1}, \mathrm{X}_{2}, \ldots, \mathrm{X}_{\mathrm{m}}\right\}$ it is necessary to compute its arithmetic mean, which is considered to be a reference value:

$$
\bar{X}=\frac{1}{m} \sum_{j=1}^{m} X_{j}
$$

Then, having determined errors of the MIs $\left\{\Delta_{1}\right.$, $\left.\Delta_{2}, \ldots, \Delta_{\mathrm{m}}\right\}$ and having compared values of these errors with maximum allowable limits for each MI there can be identified MIs whose error exceeds the allowable limits and, therefore, they should be removed from the process of measurements and be calibrated. An important requirement in the implementation of this method of comparison is to ensure the stability of the reference signal source while recording readings of all the MIs under test.

$$
\begin{aligned}
& \Delta_{12}=X_{1}-X_{2}=\Delta_{1}-\Delta_{2}=\gamma_{12}+\eta \\
& \Delta_{13}=X_{1}-X_{3}=\Delta_{1}-\Delta_{3}=\gamma_{13}+\eta \\
& \cdots \cdots \cdots \cdots \cdots \cdots \\
& \Delta_{1 j}=X_{1}-X_{j}=\Delta_{1}-\Delta_{j}=\gamma_{1 j}+\eta
\end{aligned}
$$


If an in-plant measuring system is organized in such a way that it has periodic breaks in its operation and it is inappropriate to carry out preliminary comparisons one can arrange circular comparisons of the MIs. Then, in conditions of metrological autonomy, having done consequent comparisons of MIs of the same kind, we get a matrix of comparison results [6].

After appropriate statistical processing the maximum-likelihood estimate of the comparisons is taken as the value of the "virtual" reference [4]:

$$
X^{*}=\arg \max \left\{p\left(X_{1}, \ldots X_{j} / X\right)\right\}
$$

where: $\mathrm{X}$ is a real value of a physical unit measured during comparing.

This method can also be used for comparing MIs of the same kind of unequal accuracy if one enters into the matrix weighting factors of the mutual comparisons [4].

\section{MEASUREMENT RESULTS}

When there are several groups of MIs of the same kind or they are distributed in remote areas of the measured object, their mutual comparisons can be arranged as follows. One must select the most stable MI within each group of MIs of the same kind using the results of previous operation and calibrations. Then the procedure of mutual comparisons becomes simultaneous with measurements using the most stable MI and each MI within this set of MIs. Taking as the reference value the reading of the most stable MI and having processed the measurement results there can be identified the MIs, which should be calibrated. This algorithm of providing traceability within the in-plant measurement system is confirmed by the carried out study [2] and it is obviously less expensive because it does not require any additional technical facilities. To ensure traceability of measurements in the in-plant measurement system to the units of physical quantities, which are reproducible with state standards it is sufficient to carry out the calibration of the selected most stable MIs.

It should be noted, that MIs which belong to a certain "virtual" reference are operated in a usual way as well as the rest MIs in an in-plant measuring system. However, their high metrological stability allows using them periodically to improve metrological reliability of the set of MIs in the in-plant measuring system.

The proposed approach for estimating units of physical quantities using "virtual" references allows ensuring traceability of measurements for a wide range of units of physical quantities in inplant measuring systems. In addition, it is also possible to reduce service expenses for MIs in inplant measuring systems.

\section{CONCLUSIONS}

The creation of "virtual" references in an inplant measuring system will improve the reliability of measurements and metrological reliability of MIs used in the manufacturing processes as well as will reduce costs of metrological confirmation accordance of the MIs to required metrological standards. Further studies should be carried out towards the development of efficient algorithms to process the results of mutual comparisons within the in-plant measuring system and the creation of stable industrial calibrators.

\section{Acknowledgements}

This work was supported by Doctoral Scientific Research Fund from Hubei University of Technology (No. BSQD14037).

\section{REFERENCES}

1. Andrusyak S.A., Vladimirov V.L., Dufanets G.S., Lysyi B.M., Pinchevskii A.D., and Yasenitskii E.I. Autonomous metrological security of automatic checking systems for ac voltage and current measuring facilities in the frequency range from $20 \mathrm{~Hz}$ to $100 \mathrm{kHz}$. Measurement Techniques, 31(12), 1988, 1212-1215.

2. Andria G., Cavone G., Fabbiano L., Giaquinto N. and Savino M. Automatic calibration systemfor digital instruments without built-in communication interface. Proc. XIX IMEKO World Congress Fundamental and Applied Metrology, Lisbon, Portugal 2009, 857-860.

3. Ayres D. and Blundell A. Calibrating thermometers, (www.isotech.co.uk).

4. Bezuglov D.A. and Pomortsev P.M. Estimating the unit of the physical quantity of a collective working standard from the results of intragroup comparisons by utilizing the maximum-likelihood criterion. Measurement Techniques, 44(1), 2001, 1-8.

5. Bezuglov D.A. and Pomortsev P.M. Methodology 
for increasing intercalibration interval of a group measure. Measurement Techniques, 41(11), 1998, 985-989.

6. Birch J. Benefit of legal metrology for the economy and society. A study for the International Committee of Legal Metrology, 2003.

7. Birch J. Role and impact of legal metrology for consumer protection. ISO/COPOLCO Workshop, 2012.

8. Fridman A.E. The quality of measurements. Springer-Verlag, New York, 2012.

9. Glowacz A., Glowacz A. and Glowacz Z. Recognition of monochrome thermal images of synchronous motor with the application of quadtree decomposition and backpropagation neural network. Eksploatacja i Niezawodnosc - Maintenance and Reliability, 16(1), 2014, 92-96.

10. Ibarra-Castanedo C., Gonzalez D., Klein M., Pilla M., Vallerand S. and Maldague X. Infrared image processing and data analysis. Infrared Physics and Technology 46, 2004, 75-83.

11. ISO 10012:2003 Measurement management systems - Requirements for measurement processes and measuring equipment.

12. Klein M. and Rosenberg L. Deterioration of inventory and equipment. Naval Research Logistics Quarterly, 7(1), 1960, 49-62.

13. Kochan R., Kochan O., Chyrka M., Jun Su and Bykovyy P. Approaches of voltage divider development for metrology verification of ADC. Proc. of the 2013 IEEE 7-th International Conference on Intelligent Data Acquisition and Advanced Computing Systems, Berlin, Germany, 2013, 70-76.
14. Maruda R.W., Krolczyk G.M., Feldshtein E., Pusavec F., Szydlowski M., Legutko S. and Sobczak-Kupiec A. A study on droplets sizes, their distribution and heat exchange for minimum quantity cooling lubrication (MQCL). International Journal of Machine Tools and Manufacture, 100, 2016, 81-92.

15. Morris A.S. Measurement and instrumentation principles. Butterworth-Heinemann, 2001.

16. Mykyichuk M. Measurement assurance of production quality while producing. Vyzhnytsya, 2014.

17. Mykyichuk M., Ohirko R. and Boyko T. Forecasting errors of industrial thermometers. Automation, measurements and control. Bulletin of Lviv Polytechnic National University, 500, 2004, 36-40.

18. Siemionek E. and Dziubiński M. Testing energy consumption in the trolleybus and the bus on a chosen public transport line in Lublin. Advances in Science and Technology Research Journal, 9(26), 2015, 152-153.

19. Sweet J.N. Establishment of accuracy limits and standards for comparative thermal conductivity measurements. International Journal of Thermophysics, 7(4), 1986, 743-754.

20. Tsai B.K., Gibson C.E., Murthy A.V., Early E.A., Dewitt D.P. and Saunders R.D. Heat-flux sensor calibration. NIST, 2004.

21. Winkelmans M. and Wevers M. Non-destructive testing for corrosion monitoring in chemical plants. Journal of Acoustic Emission, 20, 2002, 206-217.

22. Yatsyshyn S., Mykytyn I., Stadnyk B. and Kolodiy Z. Development of noise measurements. Part 5. miniature gas sensors and their performance. Sensors and Transducers, 154(7), 2013, 223-226. 